\title{
Information Fusion for Rural Land-Use Classification With High-Resolution Satellite Imagery
}

\author{
Wanxiao Sun, Volker Heidt, Peng Gong, and Gang Xu
}

\begin{abstract}
We propose an information fusion method for the extraction of land-use information based on both the panchromatic and multispectral Indian Remote Sensing Satellite 1C (IRS-1C) satellite imagery. It integrates spectral, spatial and structural information existing in the image. A thematic map was first produced with a maximum-likelihood classification (MLC) applied to the multispectral imagery. Probabilistic relaxation (PR) was then performed on the thematic map to refine the classification with neighborhood information. Furthermore, we incorporated edges extracted from the higher resolution panchromatic imagery in the classification. An edge map was generated using operations such as edge detection, edge thresholding and edge thinning. Finally, a modified region-growing approach was used to improve image classification. The procedure proved to be more effective in land-use classification than conventional methods based only on multispectral data. The improved land-use map is characterized with sharp interregional boundaries, reduced number of mixed pixels and more homogeneous regions. The overall kappa statistics increased considerably from 0.52 before the fusion to 0.75 after.
\end{abstract}

Index Terms-Edge extraction, high-resolution satellite imagery, image classification, information fusion, land-use classification, multispectral classification, probabilistic relaxation, region-growing algorithm.

\section{INTRODUCTION}

C ONVENTIONAL multispectral classification methods make use of spectral response of ground objects. The spectral response of ground objects within one pixel is a set of $n$ radiance measurements obtained in the various wavelength bands. This set of $n$ radiance measurements is referred to as a spectral vector in the measurement space. Because spectral responses measured by remote sensors over various features of ground objects often permit an assessment of the type and condition of the features, these responses are also referred to as spectral signatures. Usually, the spectral signatures of each class type are modeled to have multivariate normal distribution, and the parameters of such spectral signatures are estimated from training samples. Based on the spectral signatures, the spectral vector of a pixel is used to classify the pixel by using

Manuscript received November 15, 2001; revised September 17, 2002.

W. Sun is with the Department of Geography, Southern Illinois University, Carbondale, IL 62901 USA.

V. Heidt is with the Department of Geography, University of Mainz, D-55099 Mainz, Germany.

P. Gong is with the Center for Assessment and Monitoring of Forest and Environmental Resources, Department of Environmental Sciences, Policy and Management, University of California, Berkeley, CA 94720-3110 USA.

$\mathrm{G}$. $\mathrm{Xu}$ is with the Department of Geography, University of Vermont, Burlington, VT 05405 USA.

Digital Object Identifier 10.1109/TGRS.2003.810707 a classifier. This per-pixel classification approach often results in "salt-and-pepper" effects and weakly defined interregion boundaries on the final map [1]. Four major thrusts for enhancing the quality of thematic maps can be identified. The first group consists of making better use of spectral information based on an alternative spectral representation [2]. In this approach, spectral classes are represented by their spectral shapes; the spectral shape is a vector of binary features that describes the relative values between spectral bands. Wharton developed a prototype expert system to classify multispectral data on the basis of spectral knowledge [3].

The second group may be characterized as the increased use of spatial information, also known as contextual information. Five strategies can be identified in contextual classification:

1) methods based on the classification of homogeneous objects [4]-[10];

2) techniques based on probabilistic relaxation, which allow the spatial properties of a region to be used in the classification process in a logically consistent manner [11]-[16];

3) methods derived using compound decision theory and sequential compound decision theory [17]-[23];

4) frequency-based contextual classification [24]-[26] (these methods involve a data reduction algorithm to convert multispectral data into a single image followed by applying a frequency-matching algorithm in classification);

5) methods based on stochastic modeling of the distribution of classes in the scene [27], [28].

The third group is information fusion and integration with ancillary data layers. Broadly, two types of data are used in information fusion techniques: additional images (i.e., multitemporal and multisensor image data, [29]) and ground data or ancillary information [9], [30]-[33]. Conceptually, three categories of data fusion were summarized [34], i.e., data level, feature level, and decision level fusion. The fourth group involves the use of knowledge-based classification techniques. Nagao and Matsuyama [35], [36] used a knowledge base to characterize contextual and geometric constraints for the task of region labeling in multispectral imagery. This has been adapted and applied in land-use mapping with satellite imagery [37]-[40]. Other researchers have evaluated Markov-random-field-based approaches for image texture extraction in multispectral image segmentation [41].

In this study, we extracted edges from the panchromatic (PAN) image, since the PAN image has a higher spatial resolution than that of the multispectral image. Several edge detectors 
have been proposed [42]-[46]. Usually, edge detectors use firstor second-order derivatives. A texture boundary locator (TBL) calculates the texture gradient of an image, which is the local rate of change of a texture attribute [47]. Edge enhancement produces a grayscale image that carries information about the edge magnitude. A threshold operation can be used to detect edges and present them in a binary image. Globally, histogram-based adaptive thresholding is commonly used [48]-[50]. The resultant thick binary image must be thinned to produce sharp edges. A number of edge detection and edge thinning strategies exists [51]-[55]. A comprehensive review of existing thinning algorithms can be found in [56].

The objective of this study is to improve the classification accuracy of the thematic map by fusing the spectral, spatial, and structural information existing in images. For this purpose, a combined ISODATA and maximum-likelihood classification (MLC) algorithm from conventional multispectral classification, probabilistic relaxation from contextual classification, edge extraction, and region growing were selected and tested.

\section{Study Site, Data, Segmentation, AND ClasSiFiCATION SCHEME}

The study site is the county of Landau of the State Rheinland-Pfalz, Germany (Fig. 1). It is located in southwestern Germany, and lies between the Palatinate Forest "Haardt" and Rhine lowlands. This area comprises mainly an agricultural environment with a medium-sized city of Landau, a few small urban areas, and some forested areas with an area of $529 \mathrm{~km}^{2}$.

An Indian Remote Sensing Satellite 1C (IRS-1C) image acquired on 29 July 1997 was used in the study. A 1/9 subscene of PAN image and its corresponding Linear Imaging and Self Scanning Sensor III (LISS-III) image were selected. The PAN subscene contains $5143 \times 5082$ pixels, while the LISS-III subscene contains $1271 \times 1255$ pixels. The PAN data have a ground resolution of $5.8 \mathrm{~m}$, while the LISS-III data have a spatial resolution of $23.5 \mathrm{~m}$ in the green, red, and near-infrared bands.

To make it easier to compare satellite imagery with other information such as digital orthophotos and topographic maps, we transformed the original imagery to Gauss-Krueger coordinate system. As the IRS-1C PAN data has a higher resolution, it was first rectified using 28 ground control points (GCPs) measured with differential global positioning system (DGPS). The average rms error was 1.14 pixels corresponding to approximately $6.6 \mathrm{~m}$ on the ground. Using the rectified panchromatic image as the reference, the multispectral image was rectified through "image-to-image" registration. The total rms error was 0.33 pixels. These two rectifications were achieved by using a first-order polynomial and nearest neighbor resampling method.

In this study, we were only interested in the rural area, since the urban area can be better derived from another data source: Amtliches Topographisch Kartographisches Informationssystem (ATKIS). ATKIS data contain land-use information at a scale of $1: 25000$. We selected all polygons belonging to the category "urban and build up" from the ATKIS data and masked out these areas from the image. The remaining nonurban area was used in this study.

A hierarchical land-use classification system was developed. It contains three levels of land-use types: five classes at the first

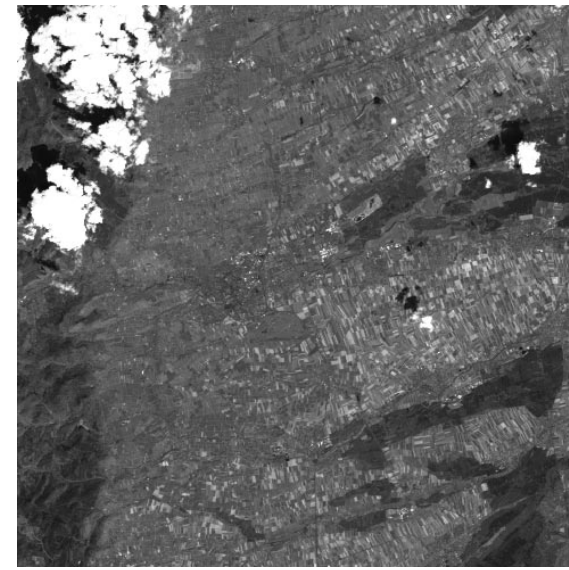

Fig. 1. IRS-1C multispectral imagery acquired on 29 July 1997, Landau, Germany, band combination 3,2,1.

TABLE I

LAND-USE CLASSIFICATION SYSTEM

\begin{tabular}{|c|c|c|}
\hline Level 1 & Level 2 & Level 3 \\
\hline \multirow[t]{6}{*}{ Agriculture } & Cropland & Barley \\
\hline & & Corn \\
\hline & & Oat \\
\hline & & Sugar beet \\
\hline & & Vine \\
\hline & & Wheat \\
\hline Grassland & Grassland & Grassland \\
\hline \multirow[t]{3}{*}{ Forest land } & Evergreen & Conifer \\
\hline & Deciduous & Deciduous \\
\hline & Mixed forest & Mixed forest \\
\hline Water & Water & Water \\
\hline Transport & Highway & Highway \\
\hline
\end{tabular}

level, seven classes at the second level and twelve classes at the third level (Table I).

\section{METHODS}

\section{A. Use of Spatial Information}

The probabilistic relaxation algorithm we adapted for generating PR map consists of four basic steps [46], [57]. First, probability calculation was made using the MLC algorithm. Assume that a digital image with a size of $M$ pixels is to be classified into $K$ classes $\left(\omega_{1}, \omega_{2}, \ldots, \omega_{K}\right)$. The class membership probabilities of pixel $i$ are defined as vector $\left[P_{i}\left(\omega_{i}\right), P_{i}\left(\omega_{2}\right), \ldots, P_{i}\left(\omega_{k}\right)\right]$, and satisfy the condition

$$
\sum_{l=1}^{K} P_{i}\left(\omega_{l}\right)=1, \quad 0 \leq P_{i}\left(\omega_{l}\right) \leq 1 .
$$


Second, calculating compatibility coefficients $r_{i j}\left(\omega_{k}, \omega_{l}\right)$ of $K$ classes from the MLC results by using

$$
r_{i j}\left(\omega_{k}, \omega_{l}\right)=\log _{10} \frac{N_{i j}\left(\omega_{k} \omega_{l}\right)}{\sum_{k=1}^{K} N_{i}\left(\omega_{k}, \omega_{l}\right) \sum_{i=1}^{K} N_{j}\left(\omega_{k}, \omega_{l}\right)}
$$

where $N_{i j}\left(\omega_{k}, \omega_{l}\right)$ is the frequency of occurrence of class $\omega_{k}$ and $\omega_{l}$ as neighbors at pixel $i$ and $j$. For each pair of neighboring pixels $i$ and $j$ and each pair of classes $\omega_{l}$ and, $\omega_{k}$ there is a compatibility measure $r_{i j}\left(\omega_{k}, \omega_{l}\right)$. The compatibility coefficients are then projected to the range $[-1,1]$ with -1 representing a strong incompatibility, 0 , neutral compatibility, to 1 , strong compatibility [44].

Third, deriving neighborhood function. It is defined as

$$
q_{i}^{(t)}\left(\omega_{k}\right)=\sum_{j=1}^{N_{b}} d_{i j} \sum_{l=1}^{K} r_{i j}\left(\omega_{k}, \omega_{l}\right) p_{j}^{(t)}\left(\omega_{l}\right)
$$

where $N_{b}$ is the number of neighbors considered for pixel $i$, $d_{i j}$ is the weight factor of neighbors, and $t$ is the number of iterations.

The new probability for pixel $i$ with class label $\omega_{k}$ at the $(t+1)$ th iteration is modified by multiplying the class probabilities by the neighborhood function. These new values are then normalized to one. Such a modification is an iterative process. Theoretically, it will not stop until no changes in class probabilities occur for all pixels. This can, however, lead up a huge number of iterations, or simply not converging. In practice, it is observed that the classification results will be improved in the first few iterations [16], [58].

\section{B. Structural Analysis Featured by Edge Extraction Techniques}

1) Edge Enhancement: The Sobel edge detector [43], the Prewitt edge detector [43], and the TBL edge detector were tested for the IRS-1C PAN image. We found that the TBL detector provides good results and is relatively robust to noise. Therefore, we used the TBL detector in the extraction of edge elements. The TBL algorithm takes into account the fact that on either side of two adjacent regions with gradual transition of the image intensities. The textural attribute is derived from the mean $\mu$ and standard deviation $\sigma$ of each $N \times N$ window of the image, where $N$ is obtained as a function of image features to be detected. In order to compute a texture gradient image, a $(2 B+1) \times(2 B+1)$ window is centered at each pixel, where $B$ is a function of the size of the region of interest and/or the range of the scene objects from the sensor. The window geometry for an arbitrary location $Z$ in an image is shown in Fig. 2. The pixels at the centers of the four sides and the corners of the window are labeled sequentially as shown in the figure. The texture gradient at a pixel is obtained as

$$
\max _{0 \leq i \leq 3}\left\{\left(\mu_{i}-\mu_{i+4}\right)^{2}+\left(\sigma_{i}-\sigma_{i+4}\right)^{2}\right\}^{\frac{1}{2}} .
$$

2) Edge Thresholding: In this study, histogram-based thresholding was used to produce a binary image, in that all edge elements have value one. The threshold is not an absolute one, but an upper percentage from the cumulative distribution function of the texture gradient image.

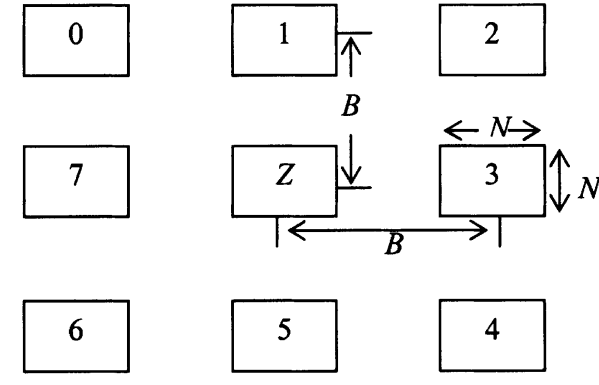

Fig. 2. Window geometry for the TBL algorithm (Bhanu et al. 1997 [47]).

\begin{tabular}{c|c|c}
\hline $\begin{array}{c}\text { P7 } \\
(i-1, j-1)\end{array}$ & $\begin{array}{c}\text { P0 } \\
(i-1, j)\end{array}$ & $\begin{array}{c}\text { P1 } \\
(i-1, j+1)\end{array}$ \\
\hline P6 & $P$ & $P 2$ \\
$(i, j-1)$ & $(i, j)$ & $(i, j+1)$ \\
\hline P5 & P4 & P3 \\
$(i+1, j-1)$ & $(i+1, j)$ & $(i+1, j+1)$ \\
\hline
\end{tabular}

Fig. 3. The $3 \times 3$ neighborhood of the pixel $P(i, j)$ (Chen-Hsu [54]).

3) Edge Thinning: The fast parallel thinning algorithm by Chen and Hsu [54] was used here, because this algorithm preserves the merits of the original such as the edge noise immunity and good effect in thinning crossing lines, and overcomes the weaknesses such as the serious shrinking and line connectivity problems. A $3 \times 3$ window is used (Fig. 3 ). The new value of pixel $P(i, j)$ at the $n$th iteration depends on its own value as well as those of its eight neighboring pixels at the $(n-1)$ th iteration, so that all image elements can be processed simultaneously. This process is divided into two subcycles. The first cycle will be executed at odd iterations while the second cycle will be executed at even iterations. A detailed explanation of the implementation of these two subcycles is given in [54].

\section{Fusion of Thematic Map and Edge Map Using A Region-Growing Algorithm}

The purpose of the fusion operation (FU) is to construct homogeneous regions over the noisy thematic map. This operation adds detailed and reliable edge information to the noisy land-use classification map to generate sharp interregion boundaries and reduce mixed pixels between them. Haralick and Shapiro [59] describe three common region-growing schemes: single-linkage region growing, hybrid-linkage region growing, centroid-linkage region growing. Pitas [60] described an efficient region-growing algorithm. This approach starts from some seed pixels representing distinct image regions and grows them, until they cover the entire image.

A modified version of Pitas' region-growing algorithm was proposed here. The image data used in the region-growing operation include the thematic map obtained from the MLC, the PR map from the probabilistic relaxation and the edge map from edge extraction. Region-growing takes three steps.

1) Automatic searching of seed pixels from the entire image. A seed pixel is the first nonedge pixel that follows directly a contour pixel. 
2) Region-growing starting with a seed pixel. This results in connected homogeneous regions. Two rules are needed, i.e., a rule describing a growth mechanism and a rule checking the homogeneity of the regions after each growth. The growth mechanism is: at each stage and for each region $R_{x}^{(s)}, x=1,2, \ldots, G$, it will be checked if there are unclassified pixels in a four-neighborhood of each edge pixel and in the eight-neighborhood of each nonedge pixel. Before assigning such a pixel $i$ to a region $R_{x}^{(s)}$, it will be checked if the region homogeneity is still valid. Two conditions are used in checking the homogeneity of the regions: 1) an edge on the edge map is reached; and 2) a class change on the PR map occurred. In other words, the algorithm makes "seed" regions grow until reaching a closed edge on the edge map or a class change on the PR map.

3) Assigning the winning class to all pixels of each connected homogeneous region. The majority class of the connected region is first computed. Class labels of each pixel in connected regions are the classes resulted from the MLC. This class is defined as the winning class, and then assigned to all pixels in the homogeneous region. These three steps are repeated until all pixels in the image have a class label. This operation results in an improved thematic map.

A software package consisting of four groups of algorithms using $\mathrm{C}$ was developed. The package comprises the MLC algorithm, PR algorithm, edge extraction techniques (the TBL edge detector, the histogram edge thresholding algorithm, and a fast parallel thinning algorithm), and a region-growing information fusion algorithm.

\section{REsult AND Discussion}

\section{A. Multispectral Classification}

A hybrid method combining ISODATA and the MLC algorithm was first performed on the three bands of the LISS-III rural imagery. For the implementation of MLC, a signature file containing the means and variance-covariance matrices of 36 spectral classes was generated from both the ISODATA clustering procedure and training samples.

Fifty spectral classes were first created from the ISODATA clustering algorithm. The 50 classes in the thematic layer were then assigned the actual class names by comparing the original image data with the individual classes. As a result, 14 specrtral classes corresponding to clearly identified conifer, deciduous, barley, cloud and shadows were obtained. Twenty-two additional spectral signatures were generated using training samples. A total number of 400 training sample areas were selected with knowledge gained from fieldwork, topographic maps, digital orthophotos, original PAN and LISS-III data, merged PAN and LISS-III data, as well as the ATKIS data. It should be noted that the fieldwork was done in 1998, one year later than the acquisition of the satellite data. For some crop types that might change from year to year, training sample selection was conducted using an agricultural database of the town of Suedliche Weinstrasse. This database records the actual crop types of fields for 1997.

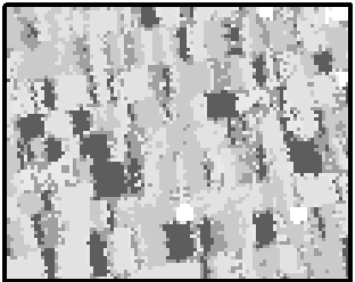

(a)

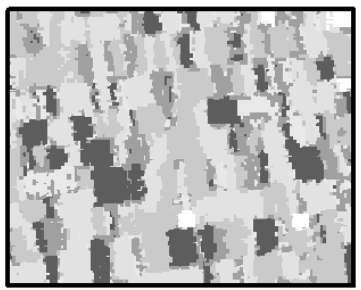

(c)

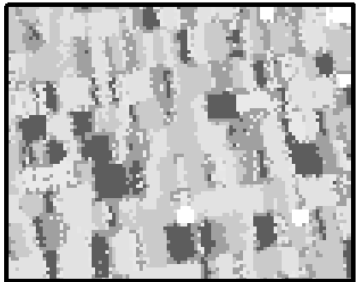

(b)

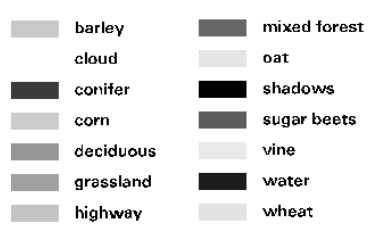

Fig. 4. Resultant maps from (a) MLC and ISODATA classification, (b) probabilistic relaxation after ten iterations, and (c) fusion of MLC map, PR map, and edge map.

This classification results in a thematic map with 36 spectral classes. These spectral classes were then grouped into 14 land-use classes according to the actual classes they represent. A subset of the MLC result is shown in Fig. 4(a). In addition to the 12 classes listed in our land-use classification system, cloud and shadows were also included. Classification accuracy and kappa statistics for each class were calculated for the MLC, PR, and region-growing fusion operation. The overall classification accuracies and kappa statistics are listed in Table II. A total of 350 test sample points were randomly selected. The number of sample points for each class is stratified to the distribution of classes. During test sample collection, it was obvious to see, at the center of sample areas, that the pixels are accurately classified with any of the three methods (MLC, PR, and FU). At the inner edges, however, the pixels are often incorrectly classified by MLC, and correctly identified by PR and FU. At the outer edges, the pixels are sometimes only correctly classified by FU.

It can be seen that cloud, wheat, sugar beet, water, barley, and mixed forest have been well classified by the MLC. Their classification accuracies are greater than $60 \%$. This is because those classes have better spectral separability. Heavy confusion occurred between vineyard and grassland because of their similar spectral responses in this season. A unique feature of the landscape of this area is the extensive fields of vineyards. Owing to different vine types, exposition, soil condition, and growing status, eight different spectral signatures of vine were first generated by using training samples. After MLC, these spectral signatures were then merged into one land-use type. Similar to vine, six spectral classes of grassland were also first trained for classification, and then merged into one land-use type. After comparing the mean plots, histograms, and statistics of signatures from vine and grassland, it was found that the two spectral classes overlapped to some extent. As a result, these two classes cannot be identified clearly, and they are mixed together. Other confusion occurred between sugar beet and grassland and grassland and forest. 
TABLE II

ClassificATION ACCURACY AND KAPPA StATISTICS COMPARISON AMONG MLC, PR, AND FU APPROACHES

\begin{tabular}{|c|c|c|c|c|c|c|}
\hline \multirow[b]{2}{*}{ Class Name } & \multicolumn{3}{|c|}{ Producers Accuracy } & \multicolumn{3}{|c|}{ Kappa statistics } \\
\hline & MLC & $\mathrm{PR}$ & $\mathrm{FU}$ & MLC & PR & FU \\
\hline barley & $60.00 \%$ & $50.00 \%$ & $86.67 \%$ & 0.6635 & 0.652 & 0.8542 \\
\hline cloud & $88.89 \%$ & $88.89 \%$ & $94.44 \%$ & 1 & 1 & 1 \\
\hline conifer & $33.33 \%$ & $79.17 \%$ & $83.33 \%$ & 0.4632 & 0.7109 & 0.8211 \\
\hline corn & $55.56 \%$ & $0.00 \%$ & $55.56 \%$ & 0.5006 & 0 & 1 \\
\hline deciduous & $55.56 \%$ & $77.78 \%$ & $80.56 \%$ & 0.5223 & 0.7067 & 0.759 \\
\hline grassland & $41.67 \%$ & $58.33 \%$ & $75.00 \%$ & 0.2653 & 0.3109 & 0.4532 \\
\hline highway & $55.56 \%$ & $61.11 \%$ & $72.22 \%$ & 0.6047 & 0.59 & 0.8023 \\
\hline mixed forest & $62.50 \%$ & $70.83 \%$ & $79.17 \%$ & 0.5974 & 0.839 & 0.8978 \\
\hline oat & $45.00 \%$ & $40.00 \%$ & $80.00 \%$ & 0.536 & 0.6465 & 0.8325 \\
\hline shadows & $50.00 \%$ & $50.00 \%$ & $83.33 \%$ & 0.4729 & 0.5388 & 0.6988 \\
\hline sugar beets & $62.50 \%$ & $75.00 \%$ & $87.50 \%$ & 0.5974 & 0.8048 & 0.8282 \\
\hline vine & $52.78 \%$ & $52.78 \%$ & $58.33 \%$ & 0.3006 & 0.2704 & 0.518 \\
\hline water & $61.11 \%$ & $72.22 \%$ & $72.22 \%$ & 0.8378 & 0.8594 & 0.9247 \\
\hline wheat & $66.67 \%$ & $76.67 \%$ & $80.00 \%$ & 0.5898 & 0.625 & 0.9159 \\
\hline $\begin{array}{l}\text { Overall } \\
\text { Classification } \\
\text { Accuracy }\end{array}$ & $55.71 \%$ & $62.00 \%$ & $77.43 \%$ & & & \\
\hline $\begin{array}{l}\text { Overall Kappa } \\
\text { Statistics }\end{array}$ & & & & 0.5188 & 0.5859 & 0.7548 \\
\hline
\end{tabular}

\section{B. Probabilistic Relaxation}

Probabilistic relaxation was performed on the rural thematic map. This operation generated the PR map. Experimental results show that after initial probabilistic relaxation, cloud, shadows, and highway tended to be overclassified. To overcome this problem, a modification of compatibility coefficients was done. Compatibility coefficients between cloud and shadows, and highway and each of the 36 signatures were set to zero or very small. This means if the class of a pixel belongs to any of the classes of cloud, shadows or highway, the class probabilities of that pixel would be influenced to a much less extent by the neighboring pixels during the relaxation process.

After visual comparison it was found that the best result was achieved after ten iterations. In cases where a pixel possessed a high probability, there was no change in class reassignment after the relaxation. Otherwise, if a pixel had low probabilities, the class of the central pixel would be replaced by the class of neighboring pixels. The probabilistic relaxation process was computationally intensive. For example, for an image size of 1.6 MB with 36 classes, each iteration took approximately one hour on a Sun Solaris UltraSparc-2 workstation.

A subset of the class map after probabilistic relaxation is shown in Fig. 4(b). A comparison of the PR map with the MLC map suggests that the probabilistic relaxation operation has resulted in a significant reduction of the "salt-and-pepper" effect in the MLC map. Furthermore, the number of mixed pixels has reduced. Consequently, regions in the image have become more homogeneous, while line elements are maintained.

Table II gives us a quantitative measurement after PR. The overall classification accuracy increased from $56 \%$ to $62 \%$, and overall kappa statistic increased from 0.52 to 0.59 . Of those classes, forest (i.e., conifer, mixed forest, and deciduous) gained most improvement, from 0.46 to $0.71,0.60$ to 0.84 , and 0.52 to 0.71 , respectively. This is because lots of isolated pixels contained in these classes were removed, and regions in the image have become more homogeneous. The classification accuracy of grassland increased from $42 \%$ to $58 \%$, whereas the classification accuracy of vine stayed unchanged $(53 \%)$ with the kappa decreased a bit. The reason is that generating homogeneous patterns is an important characteristic of PR. In this case, the vine polygons are more homogeneous than grassland polygons before PR operation. The kappa of barley also slightly decreased. Classification accuracies of cloud and shadows stayed unchanged. However, the class of "corn" was reduced to zero. This is because there are very few corn fields in the study area leading to a negative compatibility coefficient between corn and other classes.

\section{One-Pixel Width Edge Map Generation}

1) Generation of the Edge Map: The following combinations of parameters $B=1,2,3 ; N=3,5,7 ; T=15,25$, and $35 \%$ were tested. As can be seen from the combination in Fig. 5(a), thick edges are the main problem with this edge detector.

2) Generation of Pixel-Width Edge Map: The thinning operation generated many closed boundaries and resulted in a onepixel-width edge map. Results suggested that the combination of $B=1, N=3$ and $T=15 \%$ is the best [Fig. 5(b)]. As can be seen from the figure, there are a lot of isolated points and short lines that do not form connected boundaries. Some filters such as the median and Lee-Sigma filter were tested. These unwanted details have been reduced significantly by using a 


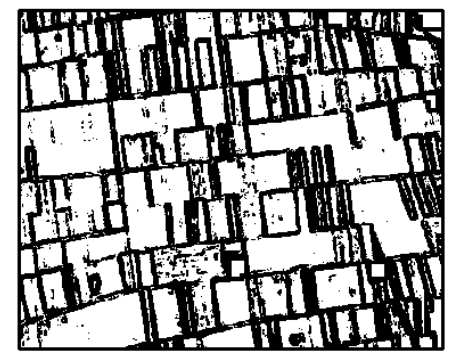

(a)

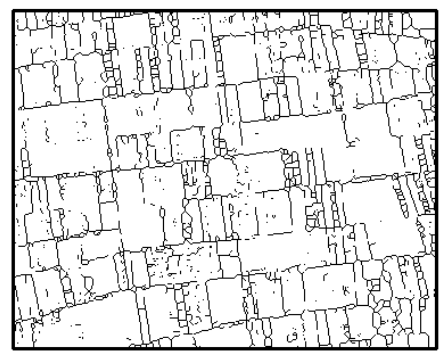

(b)

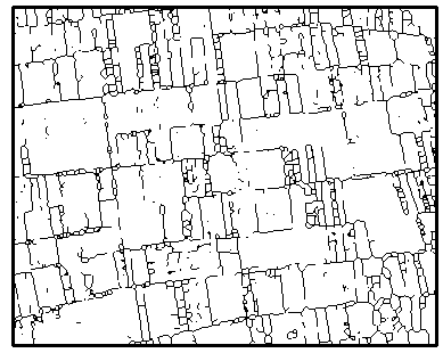

(c)

Fig. 5. Resultant maps from (a) TBL algorithm: $B=1, N=3, T=15 \%$, (b) Chen-Hsu algorithm: $B=1, N=3, T=15 \%$, and (c) edge map after using Lee-Sigma filter.

Lee-Sigma filter [Fig. 5(c)]. Lee-Sigma filter uses the average of all pixel values within the moving window that fall within the designated range of standard deviations. Field visits indicated that even field paths were detected.

\section{Fusion of Thematic Map and Edge Map Using Region-Growing Algorithm}

Three information sources were used in the fusion: 1) the MLC map, 2) the PR map, and 3) the edge map. Part of the final thematic map is shown in Fig. 4(c). The fusion of the thematic map and the edge map provides a series of closed boundaries more or less corresponding to individual fields and containing a unique class. As can be seen from the figure, important boundaries that have not been detected during the edge-detection process are obtained from the probabilistic relaxation map in a coarse manner.

The overall kappa statistics of the fusion operation increased significantly, from 0.52 to 0.75 (Table II). When calculating the absolute improvement of each land-use class comparing to that of MLC, we found that corn, conifer, wheat, mixed forest, deciduous, oat, sugar beet, and shadows contributed most to the improvement. Owing to the detected edges, land-use type "corn," which was reduced to zero after the probabilistic relaxation, is rectified. The clearly identified edges of oat result in a large improvement in classification accuracy. Wheat and sugar beet have relatively large and homogeneous fields. Their edges are the most clearly detected, and form lots of closed boundaries. The spectral variance at different locations between forest and cropland helps increasing the classification accuracy very much.

It is worthwhile to note that the kappa statistics for vine increased from 0.3 to 0.52 , and grassland from 0.27 to 0.45 . The accuracies of vine and grassland with similar spectral responses have also been improved to some extent.

\section{CONCLUSION}

A series of procedures were taken to improve land-use classification in the rural areas by making fuller use of the spatial and structural information of IRS-1C PAN imagery and the spectral information in the multispectral data. Each procedure can be implemented simply, and the integration is effective in preserving thin and edge details, which a simple filter cannot achieve. In an earlier stage of our project, we tested the majority filter after performing MLC, and the majority filter resulted in more homogeneous patterns by reducing "salt-and-pepper" noise. But the mixed-pixel problem in the interregion areas and blurred interclass boundaries still exist, and cannot be solved. The combination of data fusion, edge detection, and probability relaxation algorithms as a whole is worth documenting. In any image classification tasks, it is easy to get some results but harder to improve the accuracy. The results of this research have general implications. The combined use of multiresolution data by taking advantage of the strengths of each type of data can be extended to classification of PAN and multispectral images from Landsat ETM+, SPOT, IKONOS, and Quickbird.

The main results obtained in this study are summarized below.

1) The PR operation has resulted in a considerable reduction of the "salt-and-pepper" noise in the MLC map. Consequently, regions in the image have become more homogeneous, while line elements are maintained. However, this algorithm does not work well for classes with isolated coverage and small size.

2) The texture boundary locator algorithm is relatively robust to noise. The TBL and a fast thinning algorithm have not only produced very fine edges from the IRS-1C PAN image, but also succeeded in closing most of the edges to form field boundaries. We found that the resulting edges lent robustness to the classification system against the mixed-pixel problem by capturing detailed spatial information that is not available from the multispectral data.

3) A modified region-growing algorithm was used to fuse classification information previously obtained from the MLC, the PR, and the edge map. The fusion operation resulted in more homogeneous regions over the noisy thematic map.

4) The postprocessing image fusion strategy has proven to be particularly effective for generating sharp interclass boundaries and reducing mixed pixels between interfield boundaries. Important edges that had not been detected during edge extraction were compensated from the PR map in a coarse manner. The fusion of the thematic map 
and the edge map provides a series of closed boundaries closely corresponding to individual fields with unique classes.

\section{ACKNOWLEDGMENT}

The authors are very thankful to the Ministry of Environment, the State of Rheinland-Pfalze, Germany and the German Science Foundations for their financial support on satellite imagery and this research, availability to project relevant data, and measurement of GPS base station in the field.

\section{REFERENCES}

[1] B. Solaiman, R. K. Koffi, M. Mouchot, and A. Hillion, "An information fusion method for multispectral image classification postprocessing," IEEE Trans. Geosci. Remote Sensing, vol. 36, pp. 395-406, Mar. 1998.

[2] M. J. Carlotto, "Spectral shape classification of Landsat Thematic Mapper imagery," Photogram. Eng. Remote Sens., vol. 64, no. 9, pp. 905-913, 1998.

[3] S. W. Wharton, "A spectral-knowledge-based approach for urban landcover discrimination," IEEE Trans. Geosci. Remote Sensing, vol. 25, pp. 272-282, May 1987.

[4] P. Gong and P. J. Howarth, "The use of structural information for improving land-cover classification accuracies at the rural-urban fringe," Photogram. Eng. Remote Sens., vol. 56, no. 1, pp. 67-73, 1990.

[5] R. L. Kettig and D. A. Landgrebe, "Classification of multispectral image data by extraction and classification of homogeneous objects," IEEE Trans. Geosci. Electron., vol. GE-14, pp. 19-26, Jan. 1976.

[6] D. A. Landgrebe, "The development of a spectral-spatial classifier for earth observational data," Pattern Recognit., vol. 12, pp. 165-175, 1980.

[7] R. O. Duda and P. E. Hart, Pattern Classification and Scene Analysis. New York: Wiley, 1973.

[8] F. E. Townsand, "The enhancement of computer classifications by logical smoothing," Photogram. Eng. Remote Sens., vol. 52, pp. 213-221, 1986.

[9] R. Schowengerdt, Techniques for Image Processing and Classification in Remote Sensing. New York: Academic, 1983.

[10] H. Bischof, W. Schneider, and A. J. Pinz, "Multispectral classification of Landsat-images using neural networks," IEEE Trans. Geosci. Remote Sensing, vol. 30, no. May, pp. 482-493, 1992.

[11] A. Rosenfeld, R. A. Hummel, and S. W. Zucker, "Scene labeling by relaxation operations," IEEE Trans. Syst. Man Cybernet., vol. 6, pp. 420-433, June 1976.

[12] S. Peleg, "A new probabilistic relaxation scheme," IEEE Trans. Pattern Anal. Machine Intell., vol. PAMI-2, pp. 362-369, 1980.

[13] J. O. Eklundh, H. Yamamoto, and R. Rosenfeld, "A relaxation method for multispectral pixel classification," IEEE Trans. Pattern Anal. Machine Intell., vol. PAMI-2, pp. 72-75, 1980.

[14] O. D. Faugeras and M. M. Berthod, "Improving consistency and reducing ambiguity in stochastic labeling: An optimization approach," IEEE Trans. Pattern Anal. Machine Intell., vol. PAMI-3, pp. 412-424, 1981.

[15] J. A. Richards, D. A. Landgrebe, and P. H. Swain, "Pixal labeling by supervised probabilistic relaxation," IEEE Trans. Pattern Anal. Machine Intell., vol. PAMI-3, pp. 188-191, 1981.

[16] P. Gong and P. J. Howarth, "Performance analyzes of probabilistic relaxation methods for land-cover classification," Remote. Sens. Environ., vol. 30, pp. 33-42, 1989.

[17] G. C. Toussaint, "The use of context in pattern recognition," Pattern Recognit., vol. 10, pp. 189-204, 1978.

[18] P. H. Swain, S. B. Vardeman, and J. C. Tilton, "Contextual classification of multispectral image data," Pattern Recognit., vol. 13, no. 6, pp. 429-441, 1981.

[19] J. C. Tilton, S. B. Vardeman, and P. H. Swain, "Estimation of context for statistical classification of multispectral image data," IEEE Trans. Geosci. Remote Sensing, vol. 20, pp. 445-452, July 1982.

[20] J. Haslett, "Maximum likelihood discriminant analysis on the plane using a Markovian model of spatial context," Pattern Recognit., vol. 18, pp. 287-296, 1985.

[21] R. M. Haralick and H. Joo, "A context classifier," IEEE Trans. Geosci. Remote Sensing, vol. 24, pp. 997-1007, Nov. 1986.
[22] H. M Kalayeh and D. A. Landgrebe, "Stochastic model utilizing spectral and spatial characteristics," IEEE Trans. Pattern Anal. Machine Intell., vol. PAMI-9, pp. 457-461, 1987.

[23] N. Khazenie and M. M. Crawford, "Spatial-temporal autocorrelated model for contextual classification," IEEE Trans. Geosci. Remote Sensing, vol. 28, pp. 529-539, July 1990.

[24] P. Gong and P. J. Howarth, "Frequency-based contextual classification and grey-level vector reduction for land-use identification," Photogram. Eng. Remote Sens., vol. 58, no. 4, pp. 423-437, 1992.

[25] - "Land-use classification of SPOT HRV data using a cover-frequency method," Int. J. Remote Sens., vol. 13, no. 8, pp. 1459-1471, 1992.

[26] P. Gong, "Reducing boundary effects in a kernel-based classifier," Int. J. Remote Sens., vol. 15, no. 5, pp. 1131-1139, 1994.

[27] M. C. Zhang, R. M. Haralick, and J. B. Campbell, "Multispectral image context classification using stochastic relaxation," IEEE Trans. Syst. Man Cybernet., vol. 20, pp. 128-140, Jan. 1990.

[28] Y. Jhung and P. H. Swain, "Bayesian contextual classification based on modified $M$-Estimates and Markov random fields," IEEE Trans. Geosci. Remote Sensing, vol. 34, pp. 67-75, Jan. 1996.

[29] R. M. Haralick and L. G. Shapiro, "Survey: Image segmentation," Computer. Vis. Graph. Image Process., vol. 29, pp. 100-132, 1985.

[30] C. Tom, L. D. Miller, and J. W. Christenson, "Spatial land use inventory, modeling, and projection/denver metropolitan area with inputs from existing maps, airphotos, and Landsat imagery," National Aeronautics and Space Administration, Greenbelt, MD, NASA Tech. Memo. 79710, 1978.

[31] A. H. Strahler, "The use of prior probabilities in maximum likelihood classification of remotely sensed data," Remote Sens. Environ., vol. 10, pp. 135-163, 1980.

[32] J. R. Jensen, Digital Image Processing. Upper Saddle River, NJ: Prentice-Hall, 1986.

[33] L. Masarilla, E. H. Zahzah, and J. Desachy, "Combination of remote sensing and geocoded data for satellite image interpretation based on neural networks," in Proc. IGARSS, vol. II, 1993, pp. 725-727.

[34] "Special issue on data fusion," IEEE Trans. Geosci. Remote Sensing, vol. 37, pp. 1185-1460, May 1999.

[35] M. Nagao and T. Matsuyama, "Edge preserving smoothing," Comput. Vis. Graph. Image Process., vol. 9, pp. 394-407, 1979.

[36] — - A Structural Analysis of Complex Aerial Photographs. New York: Plenum, 1980.

[37] P. Gong and P. J. Howarth, "Land cover to land use conversion: A knowledge-based approach," in Proc. Technical Papers in Annual Conf. of American Society of Photogrammetry and Remote Sensing, vol. 4, Denver, CO, 1990, pp. 447-456.

[38] J. Ton, J. Stricklen, and A. K. Jain, "Knowledge-based segmentation of Landsat images," IEEE Trans. Geosci. Remote Sensing, vol. 29, pp. 222-232, Mar. 1991.

[39] T. Kusaka and Y. Kawata, "Hierarchical classification of Landsat TM image using spectral and spatial information," in Proc. IGARSS, 1991, pp. 2187-2190.

[40] M. Goldberg, D. G. Goodenough, M. Alvo, and G. M. Karam, "A hierarchical expert system for updating forestry maps with Landsat data," Proc. IEEE, vol. 73, pp. 1054-1063, June 1985.

[41] C. A. Therrien, "An estimation-theoretic approach to terrain image segmentation," Comput. Graph. Image Process., vol. 22, pp. 313-326, 1983.

[42] D. H. Ballard and C. M. Brown, Computer Vision. Upper Saddle River, NJ: Prentice-Hall, 1982.

[43] R. C. Gonzalez and P. Wintz, Digital Image Processing. Reading, MA: Addison-Wesley, 1987.

[44] M. James, Pattern Recognition. Oxford, U.K.: BSP Professional Books, 1987.

[45] R. Jain, R. Kasturi, and B. G. Schunck, Machine Vision. New York: McGraw-Hill, 1995.

[46] J. A. Richards, Remote Sensing Digital Image Analysis: An Introduction. Berlin, Germany: Springer-Verlag, 1994.

[47] B. Bhanu, P. Symosek, and S. Das, "Analysis of terrain using multispectral images," Pattern Recognit., vol. 30, no. 2, pp. 197-215, 1997.

[48] C. K. Chow and T. Kaneko, "Automatic boundary detection of the leftventricle from cineangiograms," Comput. Biomed., vol. 5, pp. 388-410, 1972.

[49] Y. Nakagawa and A. Rosenfeld, "Some experiments on variable thresholding," Pattern Recognit., vol. 11, pp. 5191-204, 1979.

[50] S. D. Yanowitz and A. M. Bruckstein, "A new method for image segmentation," Comput. Vis. Graph. Image Process., vol. 46, pp. 82-95, 1989. 
[51] H. Blum, "A transformation for extracting new descriptors of shape," in Proc. Symp. Models for Perception of Speech and Vision Form. Cambridge, MA: MIT Press, 1964, pp. 362-380.

[52] E. Persoon and K. S. Fu, "Shape description using fourier descriptors," IEEE Trans. Syst. Man Cybernet, vol. 7, pp. 170-179, 1977.

[53] N. J Naccache and R. Shinghal, "STPA: A proposed algorithm for thinning binary patterns," IEEE Trans. Syst. Man Cybernet., vol. SMC-14, pp. 409-418, 1984

[54] Y. S. Chen and W. H. Hsu, "A modified fast parallel algorithm for thinning digital patterns," Pattern Recognit. Lett., vol. 7, no. 2, pp. 99-106, 1988.

[55] T. Y. Zhang and C. Y. Suen, "A fast parallel algorithm for thinning digital patterns," Commun. ACM, vol. 27, pp. 236-239, 1984.

[56] L. Lam, S. W. Lee, and C. Y. Suen, "Thinning methodologies-A comprehensive survey," IEEE Trans. Pattern Anal. Machine Intell., vol. 14, pp. 869-885, Sept. 1992.

[57] S. DiZenzo, S. D. DeGloria, R. Bernstein, and H. G. Kolsky, "Gaussian maximum likelihood and contextual classification algorithms for multicrop classification," IEEE Trans. Geosci. Remote Sensing, vol. 25, pp. 805-814, Nov. 1987.

[58] J. A. Richards, D. A. Landgrebe, and P. H. Swain, "On the accuracy of pixel relaxation labeling," IEEE Trans. Syst. Man Cybernet., vol. 11, pp. 303-309, 1981

[59] R. M. Haralick and L. G. Shapiro, Computer and Robot Vision. Reading, MA: Addison-Wesley, 1992.

[60] I. Pitas, Digital Image Processing Algorithms. London, U.K.: Prentice-Hall, 1993.

Wanxiao Sun received the B.S. degree in computer-assisted cartography from Nanjing University, Nanjing, China, the M.S. degree in remote sensing and GIS from the Chinese Academy of Sciences, Nanjing, China, and the Ph.D. degree in remote sensing and GIS from University of Mainz, Mainz, Germany, in 1999.

She is currently an Assistant Professor with the Department of Geography, Southern Illinois University, Carbondale. She was a Postdoctoral Researcher in the Department of Environmental Science, Policy and Management, University of California, Berkeley, in 2001. Her present research interests focus on image classification and feature extraction algorithms applied to remotely sensed data, information fusion of multiresolution data, the integration of remote sensing and GIS techniques in environmental monitoring, management and planning, and 3-D visualization and simulation.

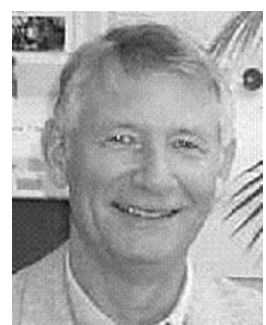

Volker Heidt received the Ph.D. degree in geography, botany, zoologym, and physics at the University of Giessen, Giessen, Germany, in 1971.

$\mathrm{He}$ is currently a Professor in the Department of Geography, University of Mainz, Mainz, Germany. He is Founder and Head of the Research Group "Ecology \& Planning" in the Department of Geography, University of Mainz and is Founder and Vice President of "RegioComun"-Institute for Strategic Regional Management. His researches focus on urban ecology and landscape planning. He is a Principal Investigator (PI) or Co-PI of several related projects. He serves as a coordinator of the ERASMUS-Intensive Program "Geography of Water," an exchange program of several European universities sponsored by the European Union.

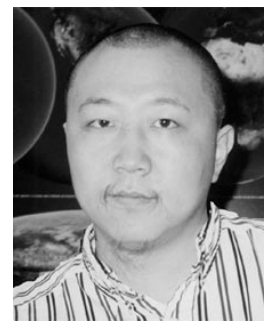

Peng Gong received the B.S. and M.S. degrees from Nanjing University, Nanjing, China, in 1984 and 1986, respectively, and the Ph.D. degree from the University of Waterloo, Waterloo, ON, Canada, in 1990.

$\mathrm{He}$ is currently a Professor in the Department of Environmental Science, Policy, and Management, University of California, Berkeley, and Co-Director of the Center for Assessment and Monitoring of Forest and Environmental Resources (CAMFER), University of California, Berkeley. He was with York University, Toronto, ON, and the University of Calgary, Calgary, AB, Canada., before joining the University of California, Berkeley, in 1994. His research interests include photoecometrics, global change monitoring, and the role of technology on society. He is an author/coauthor of over 200 papers and five books.

Dr. Gong serves as the Director of the International Institute for Earth System Science, Nanjing University, Editor-in-Chief of Geographic Information Sciences, and Editor for the International Journal of Remote Sensing.

Gang $\mathbf{X u}$ received the Ph.D. degree from the University of Mainz, Mainz, Germany.

He is currently a Visiting Assistant Professor in the Department of Geography, University of Vermont, Burlington. His research interests include economic geography, international business, spatial analysis, and tourism management. His research has been published in several scholarly journals. 